\title{
The magnetic field structure in W51A
}

\author{
A. Chrysostomou ${ }^{1}$, D. K. Aitken ${ }^{1}$, T. Jenness ${ }^{2}$, C. J. Davis ${ }^{2}$, J. H. Hough ${ }^{1}$, R. Curran ${ }^{1}$, and M. Tamura ${ }^{3}$ \\ 1 Dept of Physical Sciences, University of Hertfordshire, Hatfield, HERTS, AL10 9AB, UK \\ e-mail: a.chrysostomou, d. aitken, j.hough,r.curran@star.herts.ac.uk \\ 2 Joint Astronomy Centre, 660 N. A'ohoku Place, Hilo, HI 96720, USA \\ e-mail: t.jenness, c.davis@jach.hawaii.edu \\ 3 National Astronomical Observatory of Japan, Osawa, Mitaka, Tokyo 181-8588, Japan \\ e-mail: tamuramt@cc.nao.ac.jp
}

Received 12 September 2001 / Accepted 24 January 2002

\begin{abstract}
We present $850 \mu \mathrm{m}$ imaging polarimetry of the W51A massive star forming region performed with SCUBA on the JCMT. From the polarimetry we infer the column-averaged magnetic field direction, projected onto the plane of the sky. We find that the magnetic field geometry in the region is complicated. We compare the field geometry with $6 \mathrm{~cm}$ and CS $J=7-6$ emission and determine that the magnetic field must be relatively weak and plays a passive role, allowing itself to be shaped by pressure forces and dynamics in the ionised and neutral gases. Comparisons are drawn between our data and $1.3 \mathrm{~mm}$ BIMA interferometric polarimetry data, from which we conclude that the magnetic field must increase in importance as we move to smaller scales and closer to sites of active star formation.
\end{abstract}

Key words. ISM: magnetic fields - ISM: individual objects: W51A - stars: formation - techniques: polarimetric submillimeter

\section{Introduction}

The W51A cloud consists of a complex of Hil regions situated approximately $7-8 \mathrm{kpc}$ away. It is one of the most active and luminous sites of massive star formation in the Galaxy with all the associated signatures: masers (Leppänen et al 1998; Zhang \& Ho 1995; Genzel \& Downes 1977), HiI regions (Scott 1978), bright \& dusty cores (Jaffe et al. 1984; Genzel et al. 1982) and nearby supernova remnants (Koo \& Moon 1997a, 1997b). Radio maps show a number of HiI regions, the most luminous being G49.5-0.4 (see e.g. Mehringer 1994) which itself is dominated by the sources W51d and W51e. The former is directly associated with its infrared counterpart IRS2 (Goldader \& WynnWilliams 1994) and while W51e and the infrared source IRS1 are also closely associated, this section of the cloud is more complex. A cluster of compact HII regions are found to the east of W51e/IRS1, whose radio emission is dominated by the sources W51e2 and e1. The submillimetre emission is more closely associated with this cluster rather than with IRS1 and the peak of emission correlates with the position of W51e2 (Jaffe et al. 1984), suggesting that

\footnotetext{
Send offprint requests to: A. Chrysostomou, e-mail: a.chrysostomou@star.herts.ac.uk

* Table 2 is only available in electronic form at the CDS via anonymous ftp to cdsarc.u-strasbg.fr (130.79.128.5) or via

http://cdsweb.u-strasbg.fr/cgi-bin/qcat?J/A+A/385/1014
}

W51e is a more evolved source while W51e1 and e2 are only now undergoing star formation. This is corroborated by the detection of infalling gas associated with W51e2 by Zhang \& Ho (1997).

Here, we present $850 \mu \mathrm{m}$ polarimetry of the W51A region revealing the magnetic field structure within it. There are a number of methods which allow for mapping the magnetic field geometry, and each has its own associated problems. By far the most promising method is through polarimetry of emission from dust grains which have been aligned (it is necessarily assumed) by interaction with the local magnetic field. Previous polarimetric observations of W51A show very low polarisation (Kane et al. 1993) consistent with a non-detection. However, these measurements were made with large beams $\left(\sim 30^{\prime \prime}\right)$ within which a significant degree of depolarisation can occur. For instance, Dotson et al. (2000) present far-infrared polarimetry for W51A at $100 \mu \mathrm{m}$ taken with the KAO. Their results show low polarisation at the cores and higher degrees of polarisation in regions with more extended emission. Higher resolution and more sensitive measurements are clearly necessary to detect significant polarisation and to determine any structure in the polarisation pattern (e.g. see Lai et al. 2001 and Momose et al. 2001). In their midinfrared spectropolarimetric atlas of young stellar objects, Smith et al. (2000) measure $6 \%$ and $3 \%$ polarisation towards W51d in absorptive and emissive components, respectively, measurements made within $5^{\prime \prime}$ beams. 
The importance of magnetic fields in star formation has become increasingly more apparent in recent years as computer models have become more sophisticated (e.g. Ostriker et al. 2001). Magnetic fields are believed to play an important regulatory role during cloud collapse and accretion onto the protostar, as well as providing the driving and collimation mechanism for outflows. However, in order to comprehend the magnetic field's role properly, an empirical understanding of its behaviour is necessary.

In this paper, we present $850 \mu \mathrm{m}$ polarimetry not only of the dense cores in W51A but also of the surrounding more diffuse emission between these cores. In this fashion we investigate the relation between the magnetic field at the sites of star formation and the cold, expansive envelopes that surround them. By comparing this information with other studies we may also hope to understand what shapes the magnetic field. For instance, are the kinematics of gas and dust, as governed by gravitational collapse and the pressures within HII regions, dominant, or is the energy density in the magnetic field of sufficient magnitude to control and regulate the dynamics?

\section{Observations and reduction}

The data for W51A were observed on the night 6 October 2000 on the James Clerk Maxwell Telescope (JCMT) situated close to the summit of Mauna Kea, Hawaii. The observations were made with the SCUBA camera (Holland et al. 1999) together with a polarimetry module in the beam mounted on the entrance window of SCUBA. The polarimetry optics consist of a rotating quartz half-wave retarder ahead of a fixed wire-grid analyser (see Greaves et al. 2000 for a full description of the polarimeter). To produce a fully sampled map at $850 \mu \mathrm{m}, 16$ offset positions of the array are needed on the sky (Holland et al.). The resultant image is referred to as a "jiggle map". For these observations of W51A an east-west chop throw of $140^{\prime \prime}$ at $7.8 \mathrm{~Hz}$ was used. Complete jiggle maps were taken at specific positions of the half-wave retarder, each separated by $22.5^{\circ}$. This therefore requires 16 jiggle maps to complete one cycle of the retarder. Six such cycles were observed towards W51A.

The SURF (SCUBA User Reduction Facility; Jenness \& Lightfoot 1998) data reduction package was used to flatfield, rebin and sky noise correct the SCUBA data. Excessively noisy bolometers were identified from noise measurements conducted on the observing night and "switched-off" so that they take no further part in the data reduction. Sky subtraction with SURF is used for eliminating sky noise in SCUBA data (Jenness et al. 1998). Sky noise can be introduced, for example, by variations in the sky transparancy between the separate positions of the secondary, which make up the jiggle pattern, in a single exposure. To correct for this noise bolometers devoid of any significant flux which can be attributed to point sources, or to extended emission, are chosen. The signal from each jiggle position in these bolometers is then used to calculate and correct for any temporal variation in the sky signal. To preserve the total flux in the map, the mean background signal which is removed in this correction is then added back. Inspection of the final image showed that the weakest signal is an order of magnitude fainter than that of the diffuse emission. This weak signal being $\sim 2 \sigma$ above the sky noise, suggests that conditions were stable enough over the timescale of the chop cycle that sky background was efficiently removed by the chop. The very small, residual sky emission would have to be quite highly polarised to have any effect on our results and conclusions. With the sky noise removed, the data are then rebinned onto a $3.09^{\prime \prime}$ pixel grid, representing half the spacing of the jiggle pattern, to form the final images.

Before rebinning the images and calculating the polarisation, the main lobe instrumental polarisation (IP) is removed from each bolometer using a look-up table of elevation dependent IP values. This software also corrects for the parallactic angle at the time of observations by altering the value of the effective retarder position on the sky. Following Greaves et al. (2002) and Matthews et al. (2001), contamination due to sideband lobe instrumental polarisation is estimated to be negligible (i.e. within errors) for our data.

The $I, Q$ and $U$ Stokes parameters were computed for each cycle of the retarder using the POLPACK polarimetry data reduction software (Berry \& Gledhill 1999). For each cycle, images taken at equivalent positions of the retarder are averaged together (e.g. positions at $0^{\circ}, 90^{\circ}$, $180^{\circ}$ and $270^{\circ}$ equivalently measure the same angle on the sky). The result is a stack of four images where each pixel in the stack samples the modulated signal at angles close to $0^{\circ}, 22.5^{\circ}, 45^{\circ}$ and $67.5^{\circ}$ to which the following curve is fitted to extract the Stokes parameters (Sparks \& Axon 1999):

$I^{\prime}=\frac{t}{2}(I+\epsilon[Q \cos 2 \phi+U \sin 2 \phi])$

where $I^{\prime}$ is the expected intensity, $t$ is the throughput of the wire-grid analyser, $\epsilon$ is the polarising efficiency of the analyser, and $\phi$ is the effective retarder position angle after correction for the parallactic angle.

After combining the data from the six cycles, the Stokes parameters are binned into $3 \times 3$ pixel bins (equivalent to $9.3^{\prime \prime}$ ). Finally, the polarisation is debiassed and thresholded so that only polarisation vectors associated with positive flux $(I>0)$ and small polarisation error $(\delta P<0.5 \%)$ are included in the final data set. The bulk of our polarisation vectors lie in the $1-3 \%$ range which, when the maximum polarisation error of $\delta P=0.5 \%$ is taken into account, translates to an uncertainty for the position angles of $\Delta \theta \sim 28.6^{\circ} / \sigma_{P} \sim 15-5^{\circ}$.

Finally, flux calibration was performed by multiplying the data by a "flux calibration factor" (FCF) of $414 \mathrm{Jy} /$ beam/Volt. This FCF has been shown to be repeatably accurate to $\sim 10 \%$ for all SCUBA calibrations (Jenness et al. 2002). The value used here has been modified to account for the loss of throughput due to the polarimeter (Greaves et al. 2002). 


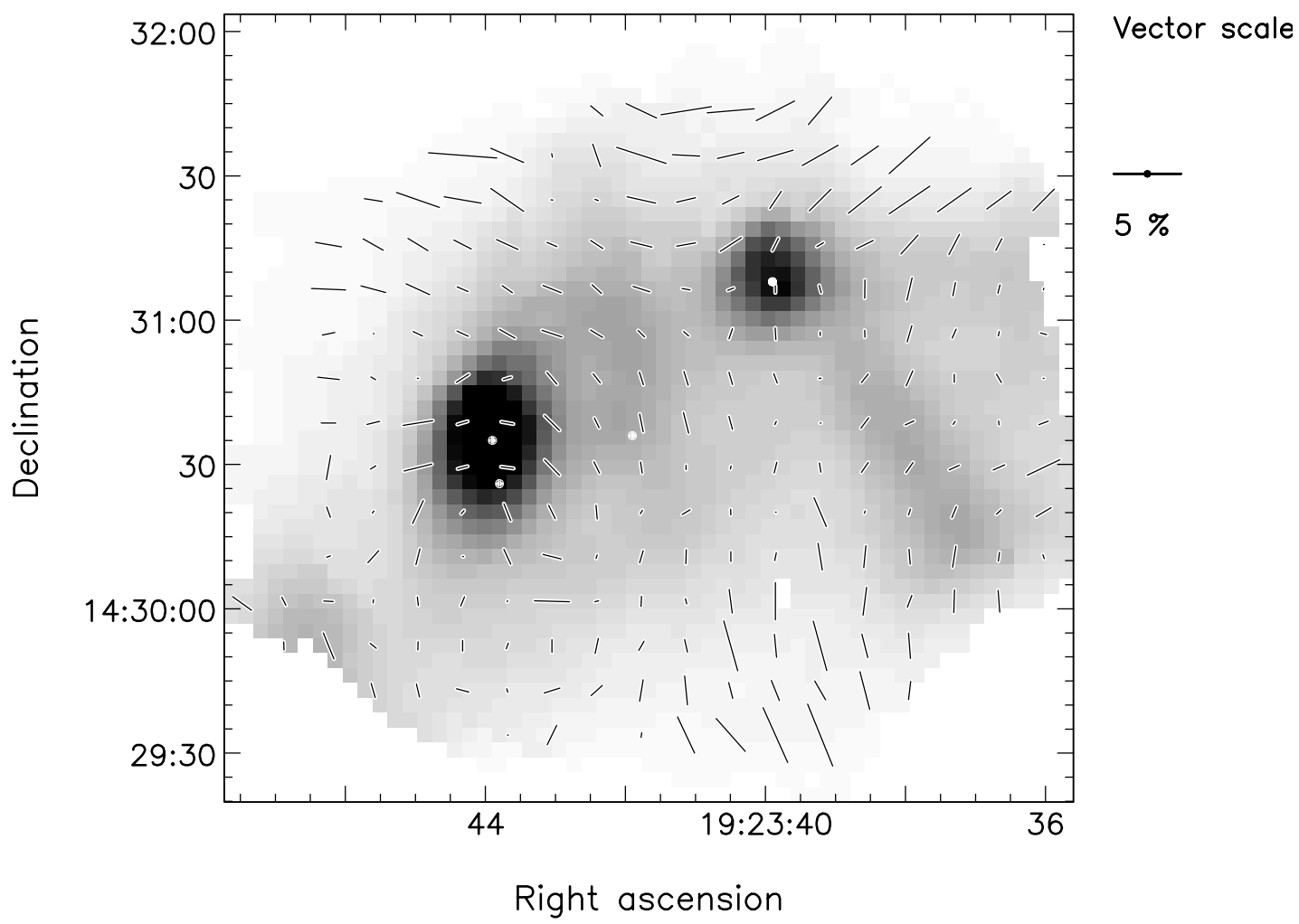

Fig. 1. SCUBA polarimetry of the W51A region. The intensity image is taken from our data and is plotted on a linear scale. The source to the east of the field is the submillimetre core associated with W51e1 and e2, and the source to the west is W51d. The polarisation vectors have been rotated by $90^{\circ}$ and plotted such that their position angles delineate the direction of the magnetic field averaged along the column and projected onto the plane of the sky. The white dots represent the positions of the brightest HiI regions in the field (see Table 1). They are, from right-to-left: W51d (IRS2), W51e (IRS1), W51e1 and W51e2 (e1 is south of e2). Right ascension and Declination are at epoch 2000.

Table 1. Brightest HiI regions and their positions in the W51A cloud. Positions obtained from the SIMBAD database.

\begin{tabular}{lll}
\hline \hline Object & RA (J2000) & Dec. (J2000) \\
\hline W51d (IRS2) & 192339.9 & +143108 \\
W51e (IRS1) & 192341.9 & +143036 \\
W51e1 & 192343.8 & +143025.9 \\
W51e2 & 192343.9 & +143034.8 \\
\hline
\end{tabular}

\section{Results and discussion}

\subsection{Submillimetre polarimetry}

The final results are tabulated in Table 2 and shown in Fig. 1. The greyscale shows the submillimetre emission which is dominated by two bright sources. To the east is the core which was mapped by Jaffe et al. (1984) at $400 \mu \mathrm{m}$ and is associated with the W51e1 and e2 compact HII regions (Mehringer 1994). The submillimetre core to the north-west is associated with the W51d HiI region and with the IR source IRS2 (Goldader \& WynnWilliams 1994). Both cores are known sites of maser activity (Genzel \& Downes 1977). Fainter and more extended emission is also seen, occupying the region between the two cores, and an extended arm of emission is seen to reach out to the south-west from IRS2.
Figure 1 shows polarisation vectors overlaid onto the submillimetre emission. These vectors have been rotated by $90^{\circ}$ to depict the column-averaged direction of the magnetic field projected onto the plane of the sky. This field direction towards the W51e1/e2 core is at a position angle of $\sim 100-110^{\circ}$, consistent with recent interferometer polarimetry at $1.3 \mathrm{~mm}$ with BIMA (Lai et al. 2001). In an atlas of mid-infrared spectropolarimetry of young stellar objects, Smith et al. (2000) observed W51d(IRS2) and deconvolved emissive and absorptive components to the spectropolarimetry. The emissive ( $\boldsymbol{E}$-vector) component was at $36^{\circ} \pm 3^{\circ}$ implying a field direction in the hotter regions at or near the HII region (there is a clear [NeII] line in the spectrum) where the $10 \mu \mathrm{m}$ emission peaks of $126^{\circ}$. The absorptive component of the $\boldsymbol{E}$-vector, and therefore equivalent to the $\boldsymbol{B}$-vector, is at a position angle of $136^{\circ}$, a difference of $\sim 40^{\circ}$ when compared to our measurement of $\sim 0^{\circ}$. Being absorptive, the dust responsible for the mid-infrared polarisation is also probably largely responsible for the submillimetre emission. This difference in the inferred magnetic field directions is most likely due to structure in the magnetic field being sampled to different optical depths at the two wavelengths (and the different beam sizes to some extent).

Scatter plots of the polarisation data (Fig. 2) show that the degree of polarisation has a maximum of $\sim 5 \%$ 



Fig. 2. Scatter and histogram plots of the polarimetry presented in Fig. 1. The top panel shows the degree of polarisation variation with the logarithm of intensity. The bottom panel shows the variation of $\sigma_{P}$, with polarisation. No points exist below the dotted line due to the data-clipping of points with polarisation errors $\delta P>0.5 \%$ (see Sect. 2).

and also that the polarisation signal-to-noise, $\sigma_{P}$, ranges up to a maximum value $\sim 20$. The top panel of Fig. 2 shows that across the region the polarisation and intensity are generally anti-correlated, a trend seen by others (e.g. Matthews et al. 2001; Davis et al. 2000). A number of physical mechanisms have been attributed to explain this effect. A decrease in grain alignment efficiency as a function of increasing depth into a cloud is often cited as the cause (e.g. Lazarian et al. 1997). This is because most known mechanisms of grain alignment are believed to decrease in efficiency at the physical conditions common to cold, dark clouds. On the other hand, small scale field structure induced by field lines being dragged and twisted under the influence of gravitational collapse, for instance, are capable of producing the depolarising effect if the telescope beam is larger than the scale lengths over which these effects occur (e.g. Aitken et al. 1997). Zhang et al. (1998) present evidence which suggest that cores within IRS1 are indeed collapsing and rotating. However, as a caveat to this it should be noted that this trend of low degrees of polarisation for high submillimetre intensities is not universal as can be seen in recent submillimetre polarimetric imaging of the NGC 7538 region (Momose et al. 2001) where the degree of polarisation remains relatively high towards the cores.

It is also worth noting that polarisation nulls can be seen to the north-east and south-west of W51e1/e2, at positions lying almost perpendicular to the field direction defined at the core. These null vectors lie at symmetric positions, that is, they are both equispaced and on the same line through the source centre. The data have been checked and there is no apparant systematic reason for these vectors to be lowly polarised apart from the fact that the polarimetry at these positions has low signal-tonoise. A physical reason may be that at these positions the field becomes wound up such that the polarisation 
drops to very low values $(P<0.5 \%)$ and the polarised intensity is averaged out within the relatively large JCMT beam. We choose to briefly discuss these features here because simulations do show that such polarisation patterns are possible for pinched and twisted field morphologies (Aitken et al. 2001) as well as for helical fields (Fiege \& Pudritz 2000), although the latter deals with linear structures and is not really applicable to discrete sources. Furthermore, such "submillimetre polarisation nulls" either side of a bright core have been previously observed towards NGC7538 IRS1 (Momose et al. 2001). In both of these cases, the field pattern appears very much like the familiar polarisation disks seen in scattered light, at nearinfrared and optical wavelengths, around sources with optically thick disks (e.g. Lucas \& Roche 1998). Higher spatial resolution observations would clearly help us to understand the physical basis behind these polarisation nulls, as we note that there are nulls present in our data at other positions in the region.

An immediately striking feature of the polarimetry as a whole is that the magnetic field threading the region does not appear uniform in direction or degree of polarisation. It is interesting to note that the polarisation vectors which are positioned central and to the south in Fig. 1 lie closely aligned to the projections of the Galactic plane and the large scale Galactic magnetic field onto the plane of the sky $\left(\mathrm{PA} \sim 20^{\circ}\right.$; Matthewson \& Ford 1971). In their study of water masers in the core of W51e2, Leppänen et al. (1998) found the masers' proper motions also aligned in this direction. Muson \& Liszt (1979) find a large scale stream of molecular gas at this same postion angle passing through the centre of the W51A region. Their CO data shows a complex of velocities with the bulk motion at $58 \mathrm{~km} \mathrm{~s}^{-1}$, the streamer velocity at $70 \mathrm{~km} \mathrm{~s}^{-1}$ and cold absorbing gas at $\sim 66 \mathrm{~km} \mathrm{~s}^{-1}$. Koo \& Moon (1997b) determined that ${ }^{13} \mathrm{CO}$ emission is associated with the streamer.

Clearly, this position angle of $\sim 20^{\circ}$ bears some significance for this region. Those vectors which closely follow this position angle, may be tracing the large-scale Galactic magnetic field which follows the Galactic plane and may also be dragged and stretched by material in the streamer. The field then becomes disrupted when it encounters the dense, star-forming cores. Towards W51e1/e2 and W51d the field appears perpendicular to this global direction, suggesting that some degree of field dragging and windingup must have occured during the initial collapse of these cores.

The arm of submillimetre emission which extends to the south-west of W51d also has the field associated with it perpendicular to the Galactic plane and the arm itself. It appears that the collapse to form this elongated structure has occured along the magnetic field lines, but this does not seem probable if those field lines are connected to field lines to the north and to the south of the structure, where the field apparantly joins the Galactic Plane. It could be that the gravitational collapse has dragged the field lines into this pattern.

\subsection{Comparison with $6 \mathrm{~cm}$ and $C S \mathrm{~J}=7-6$ emission}

We have obtained a $6 \mathrm{~cm}$ emission map of the W51A region, downloaded from the NCSA Astronomical Data Image Library. The data were originally acquired at the VLA and published by Mehringer (1994). Also, raw data from a CS $J=7-6$ raster map of the W51A region were retrieved from the JCMT archive (operated by the CADC) and reduced. Figure 3 shows contour plots of the $6 \mathrm{~cm}$ and CS emissions overlaid onto our submillimetre measurements.

The $6 \mathrm{~cm}$ map traces free-free emission from ionised gas and the eastern peak, W51e, coincides with the IRS1 source seen in the infrared. The extended structure of the $6 \mathrm{~cm}$ emission seems to correlate with the magnetic field direction quite well. The extended emission as a whole gives the impression of an envelope around the western edge of the submillimetre core and, from the polarisation pattern, the magnetic field appears to be influenced by the presence of this ionised gas. There is an extension to the north-east of W51e which follows the magnetic field lines and may form part of the molecular streamer seen by Mufson \& Liszt (1979). In all, the $6 \mathrm{~cm}$ emission possesses a degree of curvature which may be a consequence of the expansion of the HiI region being impeded by the dense submillimetre core. If one follows the inferred magnetic field direction from the north-east through to the southwest, then one finds that around the location of W51e the field is indeed curved.

Being a density tracer, the integrated intensity map of the CS $J=7-6$ emission looks very similar to the submillimetre emission. However, the contour map presented in Fig. 3 shows the integrated flux between 59 and $63 \mathrm{~km} \mathrm{~s}^{-1}$, and at these velocities, a bridge of material between the two cores becomes apparant. Channel maps of the CS data show that there exists a small velocity gradient of $\sim 3 \mathrm{~km} \mathrm{~s}^{-1}$ across the bridge. This suggests a flow along the bridge and between the two cores, although this evidence may be better interpreted in terms of a rotating structure. Proper dynamical modelling, beyond the aims and scope of this paper, is necessary for a better understanding of this structure.

The relation of the magnetic field with the material responsible for the CS $J=7-6$ emission is not as clear as for the $6 \mathrm{~cm}$ emisssion. The most intriguing feature in the CS emission is clearly the bridge structure between the two cores, a feature not pronounced in the $850 \mu \mathrm{m}$ emission. From the W51e1/e2 core, the bridge extends to the north-west. Here the magnetic field seems to lie perpendicular to the bridge, although it could easily be more influenced by the molecular streamer in the Galactic Plane (see Sect. 3.1) or the nearby W51e HiI region. Further along, the bridge then curves around to be more latitudinal; the magnetic field fans out and is now parallel with the bridge. If there really is material flow along this structure, then it may be that the magnetic field is being pulled and stretched along the bridge at this position. 


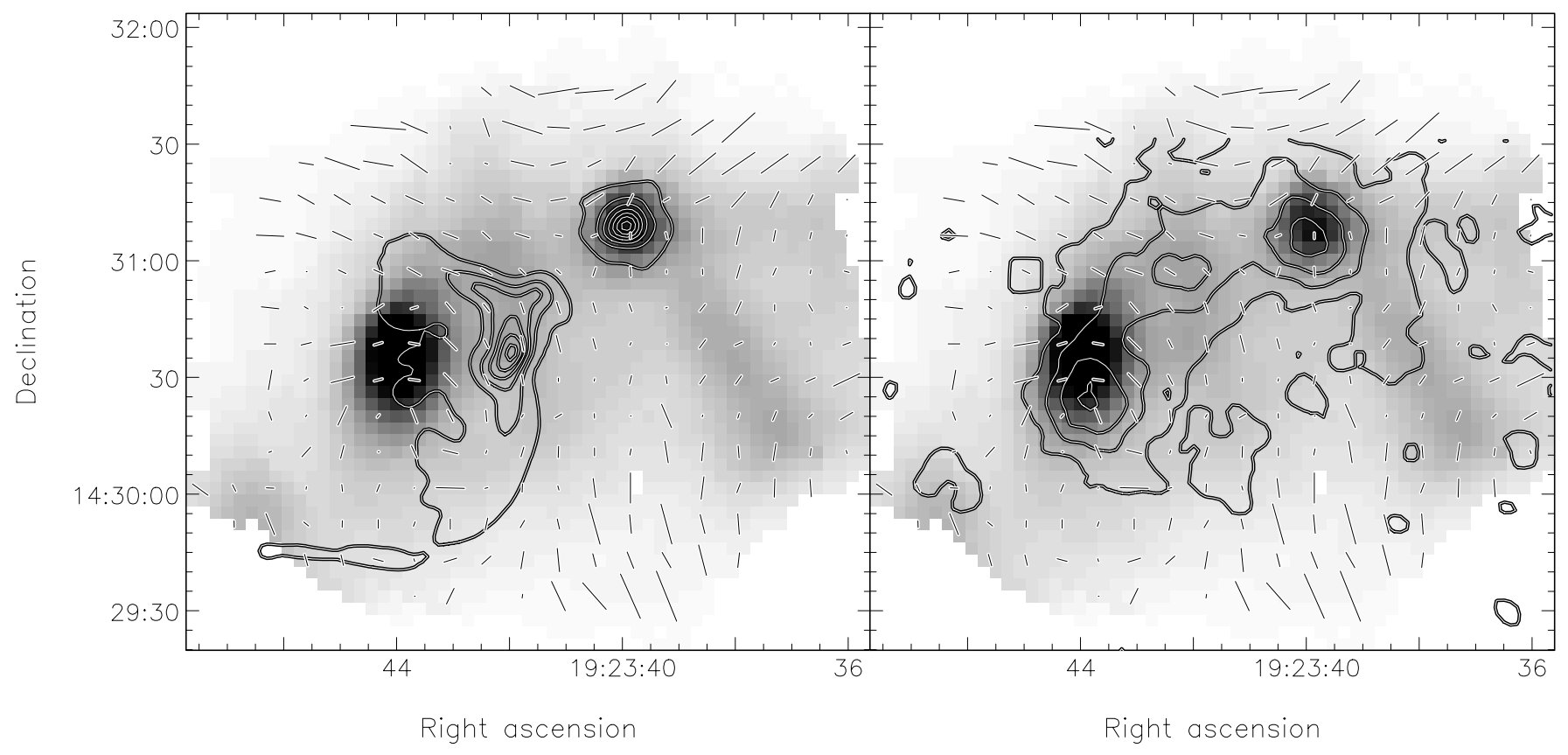

Fig. 3. Submillimetre polarimetry image of Fig. 1 overlaid with contours of $6 \mathrm{~cm}$ emission (left panel; Mehringer 1994, courtesy of ADIL) and CS $J=7-6$ emission (right panel). The presence of the W51e HII region associated with IRS1 seems to be influencing the magnetic field. The CS emission (integrated between 59 and $63 \mathrm{~km} \mathrm{~s}^{-1}$ ) reveals a bridge of dense gas between W51e1/e2 and W51d (IRS2). Once again, the magnetic field appears to be influenced by this structure, especially the northern portion of the bridge. Right ascension and Declination are at epoch 2000.

\subsection{An upper limit to the magnetic field strength}

As well as the morphology of the magnetic field, it is also important to try and obtain estimates of the magnetic field strength. Methods that have been used in the literature have included the Zeeman effect (e.g. Crutcher 1999), which gives an indication of the strength of the field component along the line of sight, and also the method of Chandrasekhar \& Fermi (1953) which gives the strength of the field component along the plane of the sky through measurement of the dispersion of polarisation position angles. The latter method is applicable to our data set but would not return sensible results due to our large beam and also because the large dispersion of position angles we find across our field renders this method futile (see Sect. 6 of Ostriker et al. 2001). However, we are able to place quite reliable upper limits to the magnetic field strength through consideration of the Virial Theorem. For a selfgravitating molecular cloud with negligible external pressure the virial equilibrium of that cloud can be written as (McKee et al. 1993; Glenn et al. 1999):

$\left|\frac{-3 G M^{2}}{5 R}\right|=2 \times 0.27 M \Delta v^{2}+0.1 B^{2} R^{3}$.

For W51e2 we use a mass of $800 M_{\odot}$ (Zhang et al. 1998), $\Delta v \sim 1 \mathrm{~km} \mathrm{~s}^{-1}$ (Young et al. 1998), and a radius for the cloud of $R \sim 3 \times 10^{15} \mathrm{~m}$, measured from the $1.3 \mathrm{~mm}$ map of Lai et al. (2001), to obtain a value of $B_{\text {up }} \sim 3.5 \mathrm{mG}$. For W51d we use a mass of $400 M_{\odot}$ (Zhang et al. 1998), $\Delta v \sim 1 \mathrm{~km} \mathrm{~s}^{-1}$ again, and a radius for the cloud of
$R \sim 5 \times 10^{15} \mathrm{~m}$, measured from the $2 \mathrm{~mm}$ map of Zhang et al. (1998), to obtain a value of $B_{\text {up }} \sim 0.6 \mathrm{mG}$.

Since we know that these clouds are in the process of collapse and forming stars, these values form useful upper limits to the value of the magnetic field strength. The value for $\mathrm{W} 51 \mathrm{e} 2$ is consistent with values obtained using the method of Chandrasekhar \& Fermi $(\sim 1 \mathrm{mG}$; Lai et al.). Crutcher (1999) presented a statistical analysis of Zeeman measurements from a number of molecular clouds (but not W51) and found line-of-sight field strengths of the order of $0.5 \mathrm{mG}$ for clouds with densities of $10^{5-6} \mathrm{~cm}^{-3}$.

\section{The magnetic field in W51A: Just how important is it?}

In Sect. 3 above, we describe how the magnetic field follows the curvature of the extended $6 \mathrm{~cm}$ emission and is also influenced by the bridge of CS $J=7-6$ emission between the two submillimetre cores. The apparant curvature of the field and the manner in which the field aligns with the velocity gradient in the CS bridge can be interpreted as evidence that the magnetic field is being shaped by the pressure and dynamics of the gas. Similar magnetic field behaviour has been seen in recent SCUBA polarimetry of NGC 7538 (Momose et al. 2001) where the blueand red-shifted outflow lobes from IRS11 are observed to have quite a high degree of curvature. The magnetic field inferred from the polarimetry follows the same curvature. However, one would expect that if the field were of sufficient strength, magnetic tension in the field lines would 
resist such action and return them back into an ordered and linear configuration.

Towards the core of the W51e1/e2 region we find that the magnetic field direction is oriented roughly perpendicular to the long axis of the extended envelope. This is clearly suggestive that the material collapsed along the field lines and then fragmented to form the cores now present. This interpretation is advocated by Lai et al. (2001) who found a very uniform field direction across the two cores (with the benefit of $3^{\prime \prime}$ resolution from their interferometry data) and interpreted this to mean that the magnetic field plays a dominant role. However, rotational motions have been detected towards the collapsing cores of W51e1 and e2 (Zhang et al. 1998), and their rotational axes are between $40-90^{\circ}$ from that of the magnetic field. This misalignment is evidence that the magnetic field has had no influence in determining the rotational axes for these clumps, evidence that their fragmentation and collapse from the common envelope has been guided according to physical parameters other than the magnetic field.

At the small scales studied by Lai et al., their reasoning may well hold true but the evidence presented here suggests that the magnetic field in W51A does not play a dominant role in determining where gas flows. Firstly, across the $2^{\prime}$ field-of-view we find a large dispersion of polarisation position angles (see Fig. 1). Recent MHD modelling of a turbulent molecular cloud (Ostriker et al. 2001) has shown that, as expected, clouds with weak fields ( $\beta \sim 1$, where $\beta$ is the ratio of the squares of the sound and Alfvén speeds and parameterises the relative strength of the magnetic field; see Eq. (3)) produce polarisation maps with vectors which are less organised and ordered than those with stronger fields $(\beta \sim 0.01)$. This is consistent with what we are observing in W51A (see Fig. 23 in Ostriker et al.).

We may analyse this further by investigating the dimensionless parameter, $\beta$. This parameter is defined as (Ostriker et al. 2001):

$\beta=\frac{c_{\mathrm{s}}^{2}}{v_{\mathrm{A}}^{2}}=\frac{c_{\mathrm{s}}^{2}}{B_{0}^{2} / 4 \pi \rho}$

where $c_{\mathrm{S}}$ is the sound speed, $v_{\mathrm{A}}$ is the Alfvén velocity, $B_{0}$ is the magnetic field strength and $\rho$ is the mass density. Using a sound speed of $c_{\mathrm{s}}^{2}=3 k T / m$, we can rewrite the $\beta$ parameter as the ratio of the gas to magnetic pressures:

$\beta=12 \pi \frac{n k T}{B_{0}^{2}}$

where $n$ is the particle number density, $T$ is the temperature and $k$ is Boltzmann's constant. Taking estimates of density and temperature from the literature we find that $n \sim 4 \times 10^{5}-10^{6} \mathrm{~cm}^{-3}$ and $T \sim 20-35 \mathrm{~K}$ (see e.g. Lai et al. 2001 and Jaffe et al. 1984). We can now write the $\beta$ parameter specifically for the W51A region as:

$\beta \sim 10^{-8} / B_{0}^{2}$
In their simulations, Ostriker et al. defined a "strong field" condition when $\beta=0.01$ which produced well correlated and ordered polarisation maps. This translates to a field strength of $B_{0} \sim 1 \mathrm{mG}$ in W51A. Conversely, their weakfield case has $\beta=1$ which translates to a field strength of $B_{0} \sim 0.1 \mathrm{mG}$.

We therefore find a consistency between our data, which infers a weak field on large scales, and that of Lai et al., who infer a strong field on smaller scales. The magnetic field has an important role to play in the W51A region as one approaches the dense cores where star formation is active. However, as one moves to the more expansive envelope and to regions between star forming cores, the magnetic field appears weaker and plays a more passive role, allowing itself to be shaped by the pressure forces and dynamics of the gas.

\section{Conclusions}

We present $850 \mu \mathrm{m}$ polarimetry of the massive star forming region in W51A. The presented data has relatively high polarimetric signal-to-noise, which allows us to infer a magnetic field structure which is not uniform across our $2^{\prime}$ field-of-view.

We have compared our data with a VLA $6 \mathrm{~cm}$ emission map as well as with a CS $J=7-6$ map obtained from the JCMT archive. The comparison shows a correlation between the magnetic field geometry and the ionised and molecular gas. This indicates that the magnetic field is shaped by the gas dynamics and not the reverse, and therefore has a relatively weak magnetic field strength (parameterised by $\beta \sim 1$ ).

However, Lai et al. (2001) have found from their $1.3 \mathrm{~mm}$ interferometer polarimetry that at smaller scales and towards the star forming cores the field lines are regular and ordered, implying that the magnetic field is comparatively strong $(\beta \sim 0.01)$ and plays a significant role in determining the gas dynamics - although evidence suggests that further fragmentation and collapse probably occurred independent of the magnetic field. Nevertheless, when one looks at larger scales and further comparisons are made between the global field morphology and the gas dynamics, this picture breaks down.

Our data has shown how important it is to attempt to correlate magnetic field structures with the physical conditions of the (ionised and neutral) gas. In this way, one can develop a better understanding of the role magnetic fields play in star formation.

Acknowledgements. The JCMT is operated by the Joint Astronomy Centre in Hilo, Hawaii on behalf of the parent organizations Particle Physics and Astronomy Research Council in the United Kingdom, the National Research Council of Canada and The Netherlands Organization for Scientific Research. The Canadian Astronomy Data Centre (CADC), is operated by the Herzberg Institute of Astrophysics, National Research Council of Canada. This research has made use of the SIMBAD database, operated at CDS, Strasbourg, France. The authors would like to thank the NCSA Astronomy Digital 
Image Library (ADIL) for providing images for this article. We acknowledge the support through software and data analysis facilities provided by the Starlink Project which is run by CCLRC on behalf of PPARC.

The authors would like to thank the referee, Dan Clemens, for carefully reading our original manuscript and providing us with useful comments which improved on it.

\section{References}

Aitken, D. K., Smith, C. H., Moore, T. J. T., et al. 1997, MNRAS, 286, 85

Aitken, D. K., Efstathiou, A., McCall, A., \& Hough, J. H. 2002, MNRAS, in press

Berry, D. S., \& Gledhill, T. M. 1999, Starlink User Note 223, Starlink Project, CCLRC

Chandrasekhar, S., \& Fermi, E. 1953, ApJ, 118, 113

Crutcher, R. M. 1999, ApJ, 520, 706

Davis, C. J., Chrysostomou, A., Matthews, H. E., Jenness, T., \& Ray, T. P. 2000, ApJ, 530, L115

Dotson, J. L, Davidson, J., Dowell, C. D., Schleuning, D. A., \& Hildebrand, R. H. 2000, ApJS, 128, 335

Fiege, J. D., \& Pudritz, R. E. 2000, ApJ, 544, 830

Genzel, R., \& Downes, D. 1977, A\&AS, 30, 145

Genzel, R., Becklin, E. E., Moran, J. M., et al. 1982, ApJ, 255, 527

Glenn, J., Walker, C. K., \& Young, E. T. 1999, ApJ, 511, 812

Goldader, J. D., \& Wynn-Williams, C. G. 1994, ApJ, 433, 164

Greaves, J. S., Jenness, T., Chrysostomou, A., Holland, W. S., \& Berry, D. S. 2000, in Imaging at Radio though Submillimeter Wavelengths, ed. J. Magnum, \& S. Radford (San Fransisco: ASP), 217, 18

Greaves, J. S., Holland, W. S., Jenness, T., et al. 2002, in preparation

Holland, W. S., Robson, E. I., Gear, W. K., et al. 1999, MNRAS, 303, 659

Jaffe, D. T., Becklin, E. E., \& Hildebrand, R. H. 1984, 279, L51
Jenness, T., Stevens, J. A., Archibald, E. N., et al. 2002, MNRAS, submitted

Jenness, T., \& Lightfoot, J. F. 1998, in Astronomical Data Analysis Software and Systems VII, ed. R. Albrecht, R. N. Hook, \& H. A. Bushouse (San Fransisco: ASP), ASP Conf. Ser., 145, 216

Jenness, T., Lightfoot, J. F., \& Holland, W. S. 1998, SPIE, 3357,548

Kane, B. D., Clemens, D. P., Barvainis, R., \& Leach, R. W. 1993, ApJ, 411, 708

Koo, B.-C., \& Moon, D.-S. 1997a, ApJ, 475, 194

Koo, B.-C., \& Moon, D.-S. 1997b, ApJ, 485, 263

Lai, S.-P., Crutcher, R. M., Girart, J. M., \& Rao, R. 2002, ApJ, accepted [astro-ph/0107322]

Lazarian, A., Goodman, A. A., \& Myers, P. C. 1997, ApJ, 490, 273

Leppänen, K., Liljeström, T., \& Diamond, P. 1998, ApJ, 507, 909

Lucas, P. W., \& Roche, P. F. 1998, MNRAS, 299, 699

Matthews, B. C., Wilson, C. D., \& Fiege, J. D. 2001, ApJ, in press

Mathewson, D., \& Ford, V. 1971, MNRAS, 153, 525

McKee, C. F., Zweibel, E. G., Goodman, A. A., \& Heiles, C. 1993, in Protostars and Planets III, ed. E. H. Levy, \& J. I. Kunine (Tucson: Univ. Arizona Press), 327

Mehringer, D. M. 1994, ApJS, 91, 713

Momose, M., Tamura, M., Kameya, O., et al. 2001, ApJ, 555, 855

Mufson, S. L., \& Liszt, H. S. 1979, ApJ, 232, 451

Ostriker, E. C., Stone, J. M., \& Gammie, C. F. 2001, ApJ, 546, 980

Scott, P. F. 1978, MNRAS, 183, 435

Sparks, W. B., \& Axon, D. J. 1999, PASP, 111, 1298

Smith, C. H., Wright, C. M., Aitken, D. K., Roche, P. F., \& Hough, J. H. 2000, MNRAS, 312, 327

Young, L. M., Keto, E., \& Ho, P. T. P. 1998, ApJ, 507, 270

Zhang, Q., \& Ho, P. T. P. 1995, ApJ, 450, L63

Zhang, Q., \& Ho, P. T. P. 1997, ApJ, 488, 241

Zhang, Q., Ho, P. T. P., \& Ohashi, N. 1998, ApJ, 494, 636 